Research Paper

\title{
Downregulation of miR-99a/let-7c/miR-125b miRNA cluster predicts clinical outcome in patients with unresected malignant pleural mesothelioma
}

\author{
Anna Truini ${ }^{1,2, *}$, Simona Coco ${ }^{1, *}$, Ernest Nadal $^{3,4,5, *}$, Carlo Genova ${ }^{1,2}$, Marco Mora ${ }^{6}$, \\ Maria Giovanna Dal Bello ${ }^{1}$, Irene Vanni ${ }^{1}$, Angela Alama ${ }^{1}$, Erika Rijavec ${ }^{1}$, Federica \\ Biello $^{1}$, Giulia Barletta ${ }^{1}$, Domenico Franco Merlo7, Alessandro Valentino ${ }^{8}$, Paola \\ Ferro $^{8}$, Gian Luigi Ravetti ${ }^{6}$, Sara Stigliani ${ }^{9}$, Antonella Vigani ${ }^{10}$, Franco Fedeli $^{8}$, \\ David G. Beer ${ }^{5}$, Silvio Roncella ${ }^{8}$ and Francesco Grossi ${ }^{1}$ \\ ${ }^{1}$ Lung Cancer Unit, Ospedale Policlinico San Martino, Genoa, Italy \\ ${ }^{2}$ Department of Internal Medicine and Medical Specialties (DIMI), University of Genoa, Ospedale Policlinico San Martino, \\ Genoa, Italy \\ ${ }^{3}$ Thoracic Oncology Unit, Department of Medical Oncology, Catalan Institute of Oncology (ICO), L'Hospitalet del Llobregat, \\ Barcelona, Spain \\ ${ }^{4}$ Molecular Mechanisms and Experimental Therapeutics Program, Bellvitge Biomedical Research Institute (IDIBELL), \\ L'Hospitalet de Llobregat, Barcelona, Spain \\ ${ }^{5}$ Section of Thoracic Surgery, Department of Surgery, University of Michigan Medical School, Ann Arbor, Michigan, USA \\ ${ }^{6}$ Department of Pathology, Ospedale Policlinico San Martino, Genoa, Italy \\ ${ }^{7}$ Research and Statistics Infrastructure, Azienda Unità Sanitaria Locale di Reggio Emilia - IRCCS, Institute for Advanced \\ Technologies and HealthCare Protocols in Oncology, Reggio Emilia, Italy \\ ${ }^{8}$ Division of Histopathology and Cytopathology, ASL5, La Spezia, Italy \\ ${ }^{9}$ UOS Physiopathology of Human Reproduction, Ospedale Policlinico San Martino, Genoa, Italy \\ ${ }^{10}$ Division of Oncology, ASL5, La Spezia, Italy \\ *These authors have contributed equally to this work
}

Correspondence to: Simona Coco, email: simona.coco@hsanmartino.it

Keywords: malignant pleural mesothelioma, unresectable tumors, miRNA signature, miR-99a/let-7c/miR-125b miRNAs cluster, prognostic biomarkers

Received: January 31, $2017 \quad$ Accepted: July 12, $2017 \quad$ Published: August 02, 2017

Copyright: Truini et al. This is an open-access article distributed under the terms of the Creative Commons Attribution License 3.0 (CC BY 3.0), which permits unrestricted use, distribution, and reproduction in any medium, provided the original author and source are credited.

\section{ABSTRACT}

Malignant pleural mesothelioma (MPM) is an aggressive tumor with a dismal overall survival (OS) and to date no molecular markers are available to guide patient management. This study aimed to identify a prognostic miRNA signature in MPM patients who did not undergo tumor resection. Whole miRNA profiling using a microarray platform was performed using biopsies on 27 unresected MPM patients with distinct clinical outcome: 15 patients had short survival (OS $<12$ months) and 12 patients had long survival (OS>36 months). Three prognostic miRNAs (mir99a, let-7c, and miR-125b) encoded at the same cluster (21q21) were selected for further validation and tested on publicly available miRNA sequencing data from 72 MPM patients with survival data. A risk model was built based on these 3 miRNAs that was validated by quantitative PCR in an independent set of 30 MPM patients. High-risk patients had shorter median OS ( 7.6 months) as compared with low-risk patients (median not reached). In the multivariate Cox model, a high-risk score was independently associated with shorter OS ( $\mathrm{HR}=3.14 ; 95 \% \mathrm{CI}, 1.18-8.34 ; \mathrm{P}=0.022)$. 


\section{Our study identified that the downregulation of the miR-99a/let-7/miR-125b miRNA cluster predicts poor outcome in unresected MPM.}

\section{INTRODUCTION}

Malignant Pleural Mesothelioma (MPM) originates from mesothelial cells that cover the pleura. The most common cause of this disease is the exposure to the asbestos fiber; indeed, MPM is predominant in areas where there are heavy or chemical industries, shipyards and oil refineries known to be activities related to asbestos usage. MPM is characterized by a long latency and its incidence is expected to increase in some industrialized countries over the next 5-10 years [1]. Long-term survival rate of patients with MPM is generally poor with a median overall survival (OS) of 9-11 months [2].

MPM can be histologically classified into three major subtypes: epithelioid, sarcomatoid and biphasic. Most MPM patients are considered not suitable for a multimodal therapeutic approach and the majority of patients are treated with palliative chemotherapy. Patients are deemed suitable for radical treatment with curative intent if the following criteria are met: epithelioid histology, absence of lymph nodal involvement, technically resectable disease, and ability to tolerate major pulmonary surgery. In Europe, multimodal therapy including induction with platinum-based chemotherapy, extra-pleural pneumonectomy (EPP) and radiation therapy is generally performed in selected patients in specialized hospitals. As this technique is associated with relevant perioperative morbidity and mortality, another surgical approach, pleurectomy with decortication (P/D), emerged as a lung-sparing procedure suitable for the radical treatment of patients with good performance status and early stage of disease [3]. Globally, while several studies have been conducted with the aim of addressing harms and benefits of surgery in MPM, its current role remains controversial, mostly due to difficulties in patient enrollment and different expertise across surgical departments; notably, a feasibility trial designed to clarify the role of $\mathrm{P} / \mathrm{D}$ (MARS2) is currently ongoing [4]. Cisplatin with pemetrexed showed longer median OS as compared with cisplatin alone and is considered the standard of care for patients with unresectable MPM [5]. In the MAPS trial, the addition of bevacizumab to cisplatin and pemetrexed demonstrated a significant increase in the OS compared with the standard treatment [6]. There is no established second-line standard of care, although single-agent regimens with gemcitabine or vinorelbine represent a suitable approach, as well as re-challenge with pemetrexed-based chemotherapy in case of good and sustained response during first-line treatment [3].

Among the MPM patients who could not undergo surgery with radical intent, there is a relatively small subgroup whose OS is greater than 3 years; therefore, taking into account this observation, we interrogated whether specific markers were able to predict prognosis and define which patients might benefit from aggressive approaches, eventually including multi-modality treatments. To date, numerous studies reported how expression level of specific miRNAs, small noncoding RNA involved in gene expression regulation, is able to precisely predict prognosis in MPM [7-12]. Recently, Kirschner and colleagues reported the first miRNA prognostic signature in surgically resected MPM patients [13]. Specifically, the authors identified 6 differentiallyexpressed miRNAs between long and short survivors in a cohort of patients who underwent surgery. However, a significant limitation of this study is represented by the low number of MPM patients who are deemed to be adequate candidates for surgery.

We hypothesized that miRNA expression profiling might identify relevant markers associated with clinical outcome in patients with unresectable MPM, which represent the majority of cases observed in clinical practice. In the present study, we first performed a miRNA microarray expression profiling using formalin-fixed paraffin embedded (FFPE) tumor biopsy samples from patients affected by MPM who did not undergo surgery and then selected miRNAs with prognostic value were tested in an independent cohort of patients by quantitative PCR (qPCR).

\section{RESULTS}

\section{Patient characteristics}

Tumor biopsy samples from patients with unresectable MPM were divided in two independent sets: training set (TS) and validation set (VS), consisting of 27 and 30 samples respectively. Clinical and demographic characteristics of patients included are shown in Table 1. There were no significant differences according to age, gender, smoking history, asbestos exposure or histopathological subtype between both sets. About half of patients in each set were considered long-term survivors.

\section{MiRNA microarray profiling yielded differentially-expressed miRNAs}

All 27 tumor samples corresponding to the TS and 4 nonmalignant pleural samples were hybridized to the miRNA microarray, however two cases (1 long survivor and 1 normal control) failed the quality control step and were excluded from the analysis (Supplementary Figure 1). Overall, the mean number of miRNAs expressed per sample was 297 and 116 miRNAs were expressed 
Table 1: Characteristics of patients included in the training set and the validation set

\begin{tabular}{|c|c|c|c|c|}
\hline \multirow{2}{*}{ Characteristics } & Training set & Validation set & All & \multirow{2}{*}{$p$-value } \\
\hline & $(n=27)$ & $(n=30)$ & $(n=57)$ & \\
\hline \multicolumn{5}{|l|}{ Age- years } \\
\hline Median (SD) & $67.9( \pm 6.5)$ & $69.5( \pm 8.5)$ & $68.7( \pm 7.6)$ & $0.540 \dagger$ \\
\hline \multicolumn{5}{|l|}{$\operatorname{Sex}-n(\%)$} \\
\hline Male & $22(81 \%)$ & $25(83 \%)$ & $47(82.5 \%)$ & \\
\hline Female & $5(19 \%)$ & $5(17 \%)$ & $10(17.5 \%)$ & $0.854 \%$ \\
\hline \multicolumn{5}{|l|}{ Smoking $-n(\%)$} \\
\hline Smokers & $18(67 \%)$ & $19(63 \%)$ & $37(65 \%)$ & \\
\hline Never smokers & $9(33 \%)$ & $9(30 \%)$ & $18(31.5 \%)$ & \\
\hline Unknown & $0(0 \%)$ & $2(7 \%)$ & $2(3.5 \%)$ & $0.391 \$$ \\
\hline \multicolumn{5}{|c|}{ Asbestos exposure $-n(\%)$} \\
\hline Yes & $22(81 \%)$ & $20(67 \%)$ & $42(74 \%)$ & \\
\hline Unknown & $5(19 \%)$ & $10(33 \%)$ & $15(26 \%)$ & $0.205 \ddagger$ \\
\hline \multicolumn{5}{|c|}{ Histological subtype $-n(\%)$} \\
\hline Epithelioid & $20(74 \%)$ & $21(70 \%)$ & $41(72 \%)$ & \\
\hline Sarcomatoid & $3(11 \%)$ & $4(13 \%)$ & $7(12 \%)$ & \\
\hline Biphasic & $3(11 \%)$ & $3(10 \%)$ & $6(10.5 \%)$ & \\
\hline Desmoplastic & $0(0 \%)$ & $2(7 \%)$ & $2(3.5 \%)$ & \\
\hline Not typified & $1(4 \%)$ & $0(0 \%)$ & $1(2 \%)$ & $0.555 t$ \\
\hline \multicolumn{5}{|l|}{ Outcome $-n(\%)$} \\
\hline Short survivor & $15(55.5 \%)$ & $16(53 \%)$ & $31(54 \%)$ & \\
\hline Long survivor & $12(44.5 \%)$ & $14(47 \%)$ & $26(46 \%)$ & $0.866 t$ \\
\hline
\end{tabular}

$\dagger$, Student's t-test; $\ddagger$, Chi-square test.

in all samples. MiRNAs with more than 30\% missing data across all samples were filtered out and a total of 259 human miRNAs were retained in the final analysis. Among the final 259 miRNAs, 91 miRNAs were found differentially expressed in 26 MPMs as compared to 3 nonmalignant pleura at p-value $<0.05$ (Mann-Whitney $\mathrm{U}$ test). Specifically, $58 \mathrm{miRNAs}$ were significantly up-regulated (fold change $>2$ ), whereas 33 were downregulated in tumor tissues (fold change $<0.5$ ), as shown in Table 2. Supervised clustering based on 64 differentially expressed miRNAs at $\mathrm{p}$-value $<0.01$ is shown in Figure 1.

\section{Hierarchical clustering yielded two major clusters associated with clinical outcome}

In the TS, hierarchical clustering analysis based on 259 miRNAs yielded two major tumor clusters (Figure 2A). There was no statistically significant difference in age, gender or histological subtype among patients classified according to these clusters (data not shown). Interestingly, patients classified in cluster 2 had significantly shorter median OS (7 months) as compared with patients categorized in cluster 1 (median OS not reached, Log-rank $\mathrm{p}=0.035$, Figure $2 \mathrm{~B}$ ).

To identify individual miRNAs significantly associated with clinical outcome, we performed classcomparison analysis among short and long survivors. We identified 35 differentially-expressed miRNAs at a t-test p-value $<0.05$ among short and long survivors that were also associated with clinical outcome in the univariate analysis (Cox regression p-value $<0.05$, Table 3 ).

\section{A 3-miRNA prognostic signature was validated using TCGA data}

In order to validate our findings and to assess the prognostic value of these 35 miRNAs in an independent dataset, we used publicly available miRNA sequencing 
Table 2: MiRNAs differentially expressed between mesothelioma tumor and nonmalignant pleura

\begin{tabular}{|c|c|c|c|c|}
\hline ID & Average MPM & Average pleura & Fold change & P-value \\
\hline hsa-miR-4486 & 0.25 & -2.87 & 8.66 & 0.0052 \\
\hline hsa-miR-4497 & 4.77 & 2.25 & 5.76 & 0.0099 \\
\hline hsa-miR-1181 & 0.71 & -1.66 & 5.17 & 0.0052 \\
\hline hsa-miR-1273e & -0.68 & -3.03 & 5.12 & 0.0081 \\
\hline hsa-miR-4465 & 3.11 & 0.77 & 5.04 & 0.0052 \\
\hline hsa-miR-4430 & 2.91 & 0.64 & 4.82 & 0.0052 \\
\hline hsa-miR-513b & 0.03 & -2.06 & 4.25 & 0.0149 \\
\hline hsa-miR-4653-3p & 2.53 & 0.45 & 4.23 & 0.0052 \\
\hline hsa-miR-1224-5p & 3.03 & 0.96 & 4.21 & 0.0052 \\
\hline hsa-miR-4462 & 0.07 & -1.94 & 4.02 & 0.0052 \\
\hline hsa-miR-4428 & 2.57 & 0.60 & 3.92 & 0.0081 \\
\hline hsa-miR-3676-5p & 5.89 & 3.95 & 3.83 & 0.0052 \\
\hline hsa-miR-6132 & 3.27 & 1.44 & 3.57 & 0.0099 \\
\hline hsa-miR-4734 & 0.20 & -1.62 & 3.51 & 0.0065 \\
\hline hsa-miR-345-5p & -1.43 & -3.23 & 3.47 & 0.0052 \\
\hline hsa-miR-4656 & 0.54 & -1.23 & 3.42 & 0.0052 \\
\hline hsa-miR-1273f & 1.14 & -0.63 & 3.42 & 0.0099 \\
\hline hsa-miR-1972 & 2.04 & 0.31 & 3.33 & 0.0065 \\
\hline hsa-miR-6075 & 0.46 & -1.27 & 3.30 & 0.0065 \\
\hline hsa-miR-4721 & 3.70 & 1.98 & 3.29 & 0.0052 \\
\hline hsa-miR-1185-2-3p & 0.49 & -1.14 & 3.10 & 0.0052 \\
\hline hsa-miR-1233-1-5p & 0.13 & -1.49 & 3.08 & 0.0081 \\
\hline hsa-miR-4515 & 0.96 & -0.63 & 3.02 & 0.0052 \\
\hline hsa-miR-197-5p & -0.40 & -1.98 & 2.99 & 0.0149 \\
\hline hsa-miR-4478 & 1.23 & -0.35 & 2.99 & 0.0052 \\
\hline hsa-miR-513a-5p & 1.27 & -0.30 & 2.97 & 0.0052 \\
\hline hsa-miR-1185-1-3p & 1.89 & 0.33 & 2.95 & 0.0052 \\
\hline hsa-miR-4695-5p & 1.18 & -0.38 & 2.95 & 0.0052 \\
\hline hsa-miR-4758-5p & -0.25 & -1.80 & 2.94 & 0.0052 \\
\hline hsa-miR-6076 & 2.01 & 0.48 & 2.89 & 0.0052 \\
\hline hsa-miR-3620-5p & 0.09 & -1.43 & 2.87 & 0.0065 \\
\hline hsa-miR-1471 & 1.59 & 0.10 & 2.82 & 0.0099 \\
\hline hsa-miR-4746-3p & 1.63 & 0.15 & 2.78 & 0.0052 \\
\hline hsa-miR-4257 & 1.42 & -0.05 & 2.78 & 0.0065 \\
\hline hsa-miR-762 & 4.21 & 2.74 & 2.76 & 0.0052 \\
\hline hsa-miR-1973 & 5.05 & 3.60 & 2.74 & 0.0052 \\
\hline hsa-miR-371a-5p & 0.59 & -0.85 & 2.71 & 0.0052 \\
\hline hsa-miR-4672 & 1.96 & 0.53 & 2.70 & 0.0081 \\
\hline hsa-miR-3940-5p & 2.89 & 1.46 & 2.69 & 0.0052 \\
\hline hsa-miR-21-5p & -1.05 & -2.41 & 2.56 & 0.0219 \\
\hline hsa-miR-494 & 6.55 & 5.19 & 2.55 & 0.0099 \\
\hline
\end{tabular}

(Continued) 


\begin{tabular}{|c|c|c|c|c|}
\hline ID & Average MPM & Average pleura & Fold change & P-value \\
\hline hsa-miR-3648 & 1.53 & 0.19 & 2.53 & 0.0081 \\
\hline hsa-miR-4322 & -0.23 & -1.56 & 2.51 & 0.0149 \\
\hline hsa-miR-3137 & 0.92 & -0.37 & 2.44 & 0.0052 \\
\hline hsa-miR-6126 & 1.94 & 0.69 & 2.39 & 0.0065 \\
\hline hsa-miR-5585-3p & 1.59 & 0.35 & 2.37 & 0.0081 \\
\hline hsa-miR-4745-5p & 1.08 & -0.09 & 2.24 & 0.0052 \\
\hline hsa-miR-3188 & 0.72 & -0.44 & 2.23 & 0.0122 \\
\hline hsa-miR-671-5p & 1.98 & 0.84 & 2.21 & 0.0378 \\
\hline hsa-miR-4728-5p & 1.73 & 0.59 & 2.20 & 0.0081 \\
\hline hsa-miR-4417 & 0.75 & -0.37 & 2.18 & 0.0065 \\
\hline hsa-miR-937-5p & 2.87 & 1.76 & 2.16 & 0.0052 \\
\hline hsa-miR-574-5p & 2.58 & 1.47 & 2.16 & 0.0450 \\
\hline hsa-miR-135a-3p & 0.55 & -0.55 & 2.14 & 0.0149 \\
\hline hsa-miR-3162-5p & 4.62 & 3.54 & 2.11 & 0.0099 \\
\hline hsa-miR-4673 & -0.49 & -1.54 & 2.08 & 0.0099 \\
\hline hsa-miR-1290 & -0.57 & -1.61 & 2.05 & 0.0099 \\
\hline hsa-miR-3917 & -0.40 & -1.41 & 2.01 & 0.0219 \\
\hline hsa-miR-4778-5p & -0.84 & 0.21 & 0.48 & 0.0264 \\
\hline hsa-miR-6090 & 6.36 & 7.42 & 0.48 & 0.0264 \\
\hline hsa-let-7i-5p & 1.89 & 2.98 & 0.47 & 0.0317 \\
\hline hsa-miR-93-5p & -1.52 & -0.34 & 0.44 & 0.0264 \\
\hline hsa-let-7e-5p & 1.11 & 2.31 & 0.44 & 0.0317 \\
\hline hsa-miR-483-5p & 0.42 & 1.65 & 0.43 & 0.0122 \\
\hline hsa-miR-378a-3p & -0.48 & 0.77 & 0.42 & 0.0181 \\
\hline hsa-let-7a-5p & 3.79 & 5.09 & 0.41 & 0.0219 \\
\hline hsa-let-7g-5p & 0.79 & 2.22 & 0.37 & 0.0181 \\
\hline hsa-let-7d-5p & 0.59 & 2.07 & 0.36 & 0.0149 \\
\hline hsa-miR-151a-5p & -1.33 & 0.25 & 0.34 & 0.0099 \\
\hline hsa-miR-125b-5p & 1.91 & 3.60 & 0.31 & 0.0219 \\
\hline hsa-miR-342-3p & -1.51 & 0.20 & 0.31 & 0.0099 \\
\hline hsa-miR-5703 & 1.10 & 2.81 & 0.30 & 0.0081 \\
\hline hsa-miR-199a-3p & 0.42 & 2.14 & 0.30 & 0.0264 \\
\hline hsa-miR-320b & 0.44 & 2.18 & 0.30 & 0.0052 \\
\hline hsa-miR-630 & 1.66 & 3.42 & 0.30 & 0.0081 \\
\hline hsa-miR-23a-3p & 1.22 & 3.06 & 0.28 & 0.0052 \\
\hline hsa-miR-15b-5p & 0.38 & 2.23 & 0.28 & 0.0181 \\
\hline hsa-miR-199a-5p & -1.50 & 0.43 & 0.26 & 0.0378 \\
\hline hsa-miR-25-3p & -1.06 & 0.93 & 0.25 & 0.0181 \\
\hline hsa-miR-320d & 0.14 & 2.16 & 0.25 & 0.0052 \\
\hline hsa-miR-195-5p & -0.88 & 1.19 & 0.24 & 0.0264 \\
\hline hsa-miR-92a-3p & -1.32 & 0.75 & 0.24 & 0.0264 \\
\hline hsa-miR-320e & -0.44 & 1.66 & 0.23 & 0.0052 \\
\hline
\end{tabular}

(Continued) 


\begin{tabular}{lcccc}
\hline ID & Average MPM & Average pleura & Fold change & P-value \\
\hline hsa-miR-320c & 1.26 & 3.54 & 0.21 & 0.0081 \\
hsa-let-7b-5p & 3.88 & 6.26 & 0.19 & 0.0052 \\
hsa-let-7c & 1.79 & 4.25 & 0.18 & 0.0065 \\
hsa-miR-22-3p & -1.27 & 1.42 & 0.16 & 0.0099 \\
hsa-miR-99a-5p & -1.19 & 1.69 & 0.14 & 0.0219 \\
hsa-miR-1260b & -0.98 & 2.09 & 0.12 & 0.0052 \\
hsa-miR-150-5p & -1.43 & 1.77 & 0.11 & 0.0099 \\
hsa-miR-451a & 0.13 & 5.10 & 0.03 & 0.0052 \\
\hline
\end{tabular}

List of the 91 miRNAs found differentially expressed between 26 MPMs and 3 nonmalignant pleura (p-value $<0.05$ ) by microarray analysis. Specifically, 58 miRNAs were significantly up-regulated (fold change $>1.5$ ), whereas 33 were down-regulated in tumor tissues (fold-change $<0.5$ )

data from 72 MPM patients deposited in TCGA. Only 12 miRNAs remained statistically associated with OS (Table 3 and Supplementary Table 1). Interestingly, the top 3 prognostic miRNAs (miR-99a, let-7c, and miR125b) were structurally associated by their genomic location on the long arm of chromosome 21 (21q21.1) and selected for further validations. In the TS, patients with low expression of miR-99a, let-7c, and miR-125b had significantly shorter median OS $(2.9,2.9$ and 3.4 months, respectively) as compared to patients with high expression of these miRNAs (median OS not reached, Figure 3A). Accordingly, in the TCGA dataset, patients with low expression of miR-99a, let-7c, and miR-125b had significantly shorter median OS (10.9 months for all three markers) as compared to patients with high expression (19.7, 19.7, and 24.1 months, Figure 3B).

The expression of miR-99a, let-7c, and miR-125b was assessed using qPCR in the TS and a close correlation was observed among the expression values generated by microarray and qPCR (Pearson $\mathrm{r}$ coefficient: 0.55; 0.71 and 0.42 respectively, Supplementary Figure 2). In order to explore the hypothetical biological effect of these 3 miRNAs, we carried out a pathway enrichment analysis based on the validated gene targets of miR-99a, let-7c, and miR-125b. This analysis revealed that these miRNAs are significantly associated with pathways relevant in mesothelioma such as cell cycle, pluripotency of stem cells, HIF-1, ErbB and AMPK signaling pathways (Supplementary Table 2).

\section{Validation of a miRNA prognostic signature by qPCR in an independent cohort}

In the validation set, we observed that miR99a, let-7c, and miR-125b expression did not correlate with the histological type of mesothelioma (MannWhitney $p>0.05$ ), whereas their expression values were significantly higher in long-surviving patients (MannWhitney $p<0.05$, Supplementary Figure 3).
A miRNA signature was built based on the expression of miR-99a, let-7c, and miR-125b by calculating a risk score based on the sum of the expression of these miRNAs in the VS, weighted by the corresponding regression coefficients $(\beta)$ derived from the Cox regression analysis in the TS, as previously reported [14]. The median risk score value was used as cutoff to classify the patients into high-risk or low-risk. In this VS, patients with a high-risk score had a significantly shorter median OS (7.6 months; $95 \%$ confidence interval, CI, 4.4-10.9) as compared with low-risk patients (median not reached, log-rank $\mathrm{p}=0.042$, Figure 4 ). The OS rates at 24 months for high- and low-risk patients were $27.7 \% \pm$ 11.4 and $66.7 \% \pm 11.2$, respectively. In the multivariate Cox model (adjusted by age and histological subtype) a high-risk score remained as an independent prognostic marker for OS (Hazard Ratio (HR) $=3.14$; 95\% CI, 1.18 8.34; $\mathrm{p}=0.022$ ). The prognostic value of these 3 miRNAs (miR-99a, let-7c, and miR-125b) encoded at the 21q21 was therefore validated in an independent set of MPM.

\section{Copy number analysis of miR-99a/let-7c/miR- 125b locus}

Copy number variation (CNV) analysis of the miR99a/let-7c/miR-125b locus (21q21.1) was performed using droplet digital PCR (ddPCR) on 44 out of 57 patients enrolled (16/27 TS and 28/30 VS) divided as follows: 17 long survivors (LS) and 27 short survivors (SS) patients. In particular, the miR-99a/let-7c/miR$125 \mathrm{~b}$ locus data was normalized using two genes: RNA binding motif protein 11 (RBM11) targeting the centromere locus on chromosome 21 (to discern 21 monosomy) and adaptor-related protein complex 3, beta 1 subunit $(A P 3 B 1)$ mapping on $5 \mathrm{q} 14.1$. The $\mathrm{CNV}$ analysis reported 21q21.1 locus mean values in the two MPM sub-populations as follows: 1.91 (range: 1.622.27) and 1.88 (range: 1.19-2.31) in LS and SS patients, respectively (Supplementary Figure 4). In particular, two 
SS patients showed a copy number ratio lower than 1.5 , however when data were normalized based on RBMII that targets the centromere, the obtained copy number ratio was diploid (1.97 and 2.35). These results suggested a potential imbalance between the two chromosomes (21 monosomy or 5 extra-copies) rather than a loss of a copy of the locus.

\section{DISCUSSION}

MiRNA expression profiling represents a powerful tool for identifying prognostic markers in cancer, including
MPM [10]. However, to the best of our knowledge, no miRNA signature has ever been identified for predicting prognosis in unresected MPM patients. Toward this aim, in the present study we screened the whole miRNA profile in a cohort of 26 MPM patients who did not undergo radical surgery, in order to identify novel prognostic markers. The analysis identified 46 miRNAs differentially expressed in MPM specimens, some of which had been previously reported in MPM, such as downregulation of let-7 in MDM2 positive MPM tumors [15], and low expression of miRNA23a-3p in a subgroup of MPM long survivors after surgery [13].
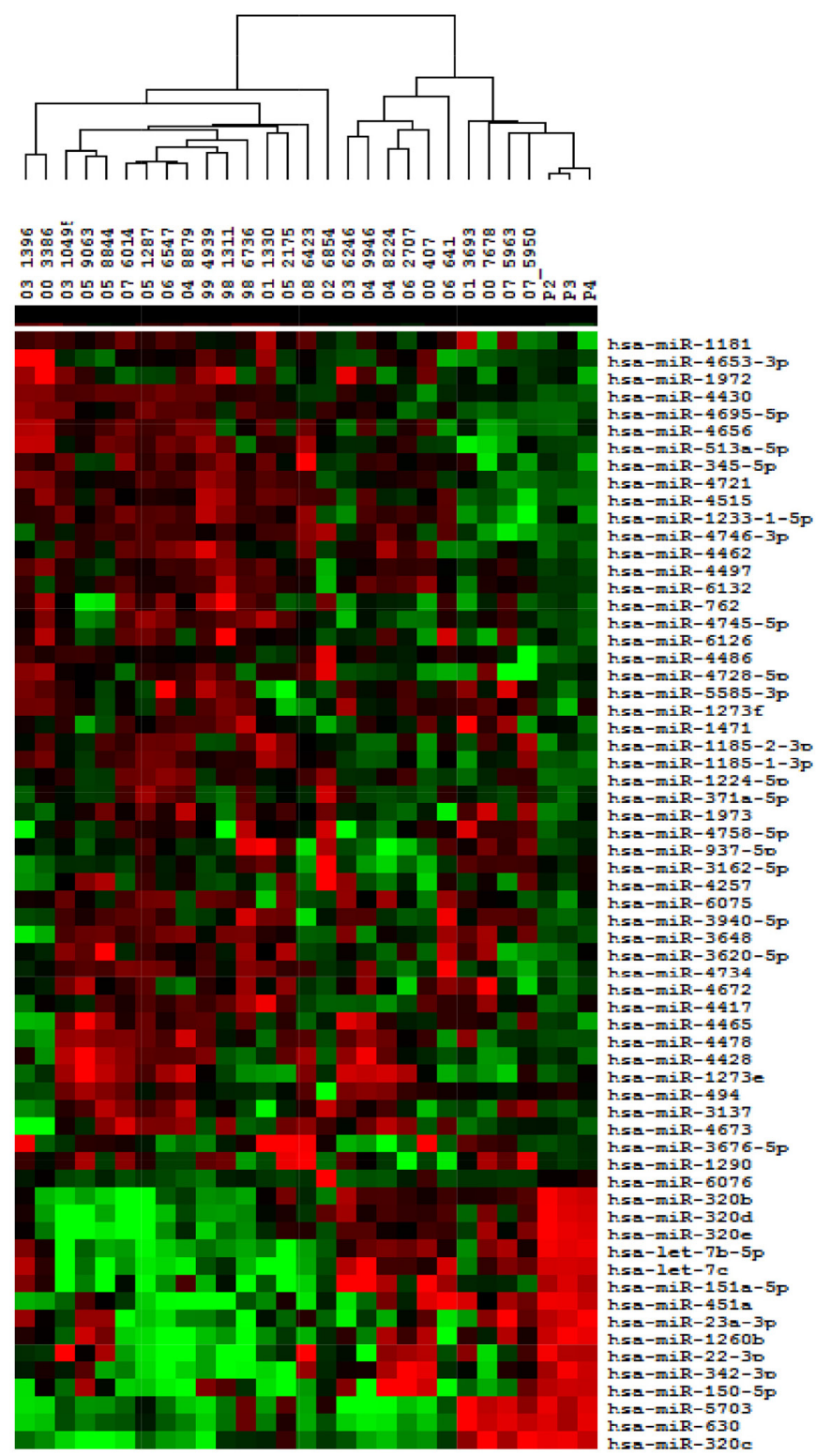

Figure 1: Supervised clustering of differentially expressed miRNAs among mesothelioma and nonmalignant pleural samples. Samples (26 MPM tumors and 3 nonmalignant pleura) are depicted in columns and miRNAs in rows. Substantially elevated (red) or decreased (green) expression of the miRNAs is observed for individual tumors. Samples and arrays were mean-centered, centroid linkage clustering using Cluster v.3.0 and visualization using Tree-View software. 
In order to investigate miRNAs with prognostic value, the 26 MPM patients were divided based on whether they had short or long survival. Class-comparison analysis of miRNA expression among short and long survivors yielded 35 differentially-expressed miRNAs, and 32 out of 35 were significantly associated with clinical outcome in the univariate analysis ( $p$-value $<0.05$ ). The prognostic value of 12 miRNAs out of these 35 was validated in an independent dataset composed of 72 MPM patients using publicly available miRNA sequencing data from TCGA.

Notably, the top three prognostic miRNAs (miR99a, let-7c, and miR-125b) among those 12 validated miRNAs, were structurally associated by their genomic location on the long arm of chromosome 21 (21q21.1).

These 3 miRNAs might play a tumor suppressor role, as they have been found to be down-regulated in distinct tumor types. In particular, their down-modulation has been described in lung cancer linked to a homozygous deletion at 21q21.1 [16], although in our cohort of samples we did not find any CNVs at the miR-99a/let-7c/miR$125 \mathrm{~b}$ cluster. Interestingly, miR-99a is among the most frequently down-regulated miRNAs in cancer, and its low expression has been linked to increased proliferation in different tumor types such as head and neck carcinoma and cervical carcinoma, as well as lung cancer, breast cancer and melanoma [17-20]. Likewise, let-7c is downregulated in a variety of cancer types such as lung and prostate cancer, melanoma and Wilms' tumors; indeed, lack of let-7c expression is associated with increased cell proliferation in both prostate and lung cancer [21, 22]. Similarly, low expression of miR-125b has been described in epithelial ovarian cancer and oral cavity squamous cell carcinoma [23, 24]. In addition, two recent studies showed concomitant downregulation of the miR-99a/ let-7c/miR-125b cluster both in cholangiocarcinoma and prostate cancer $[25,26]$. In particular, downregulation of this cluster has been linked to inflammation through the activation of IL6/STAT3 pathway [26]. Association between inflammation and MPM has already been investigated, and evidence suggests that activation of IL6 plays a role in cell proliferation and chemo-resistance $[27$, 28]. A further potential demonstration of the role of the $\mathrm{miR}-99 \mathrm{a} / \mathrm{let}-7 \mathrm{c} / \mathrm{miR}-125 \mathrm{~b}$ cluster as a tumor suppressor might be found in patients affected by Down syndrome, a genetic disorder characterized by chromosome 21 trisomy [29]. In this regard, Forés-Martos and colleagues hypothesized that the low incidence rate of solid tumors in subjects with Down syndrome might be related to the gain of copies of this miRNA cluster, located on chromosome 21 [19].

A miRNA signature was built based on the expression of miR-99a, let-7c, and miR-125b, and tested in an independent set of MPM samples in which patients were classified as high-risk or low-risk according to their
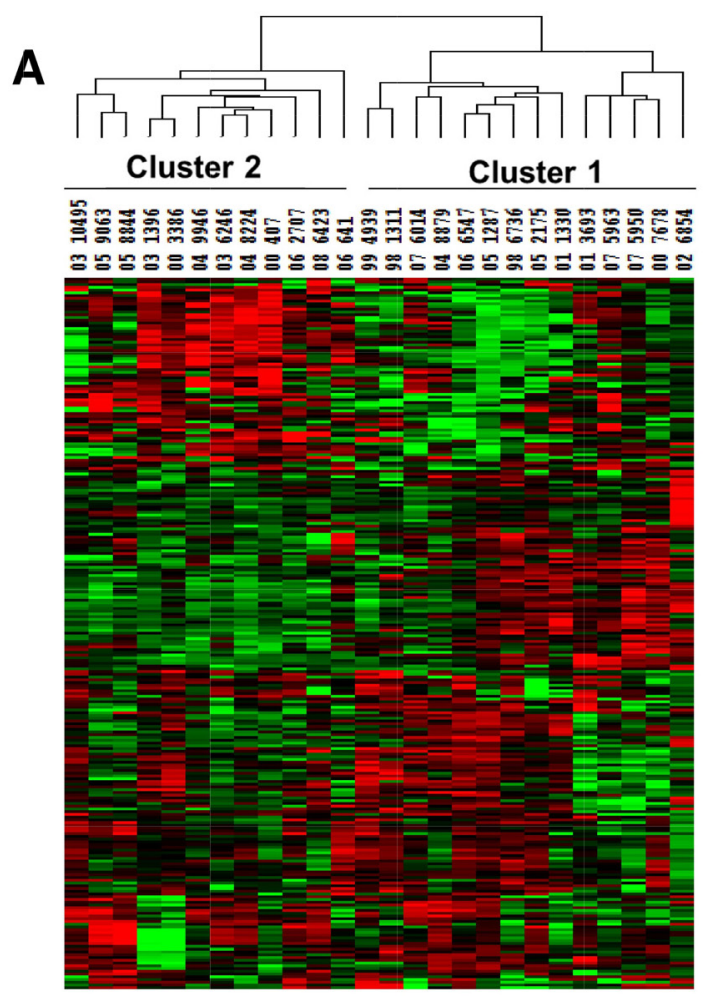

B

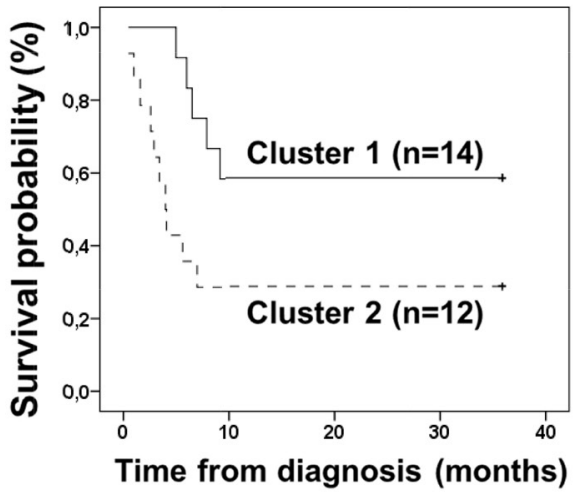

Figure 2: Unsupervised hierarchical clustering of miRNA expression in mesothelioma tumors. (A) Two major clusters of tumors were identified by unsupervised clustering analysis based on 259 miRNAs expressed in all tumors. (B) Kaplan-Meier plot of overall survival according to the cluster subgroups. 
Table 3: MiRNAs significantly associated with survival

\begin{tabular}{|c|c|c|c|c|c|c|c|}
\hline ID & Average SS & Average LS & ttest p-value & Fold Change & Cox HR & $\underset{\text { p-value }}{\text { Cox }}$ & $\begin{array}{l}\text { TCGA datase } \\
\text { validation }\end{array}$ \\
\hline miR-99a-5p & -2.07 & -0.15 & 0.0031 & 0.26 & 0.42 & 0.0014 & yes \\
\hline let-7c & 1.28 & 2.37 & 0.0066 & 0.47 & 0.32 & 0.0014 & yes \\
\hline miR-125b-5p & 1.31 & 2.62 & 0.0084 & 0.40 & 0.41 & 0.0010 & yes \\
\hline miR-26b-5p & -1.35 & -0.13 & 0.0070 & 0.43 & 0.45 & 0.0022 & yes \\
\hline miR-371a-5p & 0.79 & 0.36 & 0.0120 & 1.35 & 42.57 & 0.0010 & yes \\
\hline miR-23b-3p & -0.28 & 1.00 & 0.0201 & 0.41 & 0.53 & 0.0036 & yes \\
\hline miR-107 & -0.66 & 0.01 & 0.0275 & 0.63 & 0.27 & 0.0040 & yes \\
\hline miR-26a-5p & 0.06 & 1.02 & 0.0370 & 0.51 & 0.50 & 0.0041 & yes \\
\hline miR-30b-5p & -2.13 & -1.10 & 0.0126 & 0.49 & 0.46 & 0.0045 & yes \\
\hline miR-1185-1-3p & 2.07 & 1.69 & 0.0107 & 1.30 & 11.73 & 0.0050 & yes \\
\hline $\operatorname{miR}-29 c-3 p$ & -1.21 & -0.26 & 0.0497 & 0.52 & 0.56 & 0.0120 & yes \\
\hline $\operatorname{miR}-342-3 p$ & -1.82 & -1.14 & 0.0333 & 0.62 & 0.40 & 0.0232 & yes \\
\hline miR-652-5p & -0.84 & -1.28 & 0.0165 & 1.36 & 17.49 & 0.0010 & opposite \\
\hline miR-1236-5p & 0.69 & 0.37 & 0.0010 & 1.25 & 52.14 & 0.0020 & no \\
\hline miR-130a-3p & -0.37 & 1.08 & 0.0018 & 0.37 & 0.40 & 0.0010 & no \\
\hline miR-937-5p & 3.09 & 2.61 & 0.0023 & 1.40 & 12.21 & 0.0007 & no \\
\hline miR-1224-5p & 3.25 & 2.78 & 0.0033 & 1.39 & 35.63 & 0.0010 & no \\
\hline miR-197-5p & 5.23 & 4.84 & 0.0054 & 1.31 & 11.85 & 0.0018 & no \\
\hline $\operatorname{miR}-25-3 p$ & -1.43 & -0.63 & 0.0054 & 0.57 & 0.13 & 0.0017 & no \\
\hline let-7d-5p & 0.14 & 1.11 & 0.0085 & 0.51 & 0.23 & 0.0002 & no \\
\hline miR-642a-3p & 5.53 & 5.11 & 0.0085 & 1.34 & 22.97 & 0.0020 & no \\
\hline $\mathrm{miR}-320 \mathrm{e}$ & -0.71 & -0.13 & 0.0142 & 0.67 & 0.27 & 0.0094 & no \\
\hline $\operatorname{miR}-3137$ & 1.10 & 0.70 & 0.0144 & 1.33 & 17.53 & 0.0020 & no \\
\hline $\operatorname{miR}-16-5 p$ & 1.33 & 2.28 & 0.0153 & 0.52 & 0.2917 & 0.0002 & no \\
\hline miR-151a-5p & -1.71 & -0.88 & 0.0155 & 0.57 & 0.34 & 0.0030 & no \\
\hline $\operatorname{miR}-3162-5 p$ & 4.92 & 4.27 & 0.0195 & 1.57 & 14.91 & 0.0020 & no \\
\hline miR-1229-5p & 3.40 & 3.02 & 0.0289 & 1.30 & 6.37 & 0.0070 & no \\
\hline let- $7 b-5 p$ & 3.60 & 4.21 & 0.0302 & 0.66 & 0.24 & 0.0023 & no \\
\hline miR-939-5p & 3.06 & 2.72 & 0.0329 & 1.27 & 17.76 & 0.0014 & no \\
\hline let-7a-5p & 3.49 & 4.15 & 0.0373 & 0.63 & 0.33 & 0.0049 & no \\
\hline miR-671-5p & 2.31 & 1.61 & 0.0379 & 1.62 & 7.4268 & 0.0117 & no \\
\hline $\operatorname{miR}-15 b-5 p$ & 0.04 & 0.77 & 0.0384 & 0.60 & 0.40 & 0.0075 & no \\
\hline miR-1185-2-3p & 0.65 & 0.31 & 0.0394 & 1.27 & 7.61 & 0.0130 & no \\
\hline let-7f-5p & 2.35 & 3.01 & 0.0446 & 0.64 & 0.31 & 0.0076 & no \\
\hline $\operatorname{miR}-22-3 p$ & 0.85 & 1.65 & 0.0469 & 0.58 & 0.47 & 0.0232 & no \\
\hline
\end{tabular}

List of miRNAs significantly associated with clinical outcome in the training set. Fold-change was calculated as mean value in short survivors (SS) in respect to long survivors (LS). The last column shows whether the prognostic value of each miRNA was validated using TCGA data. 


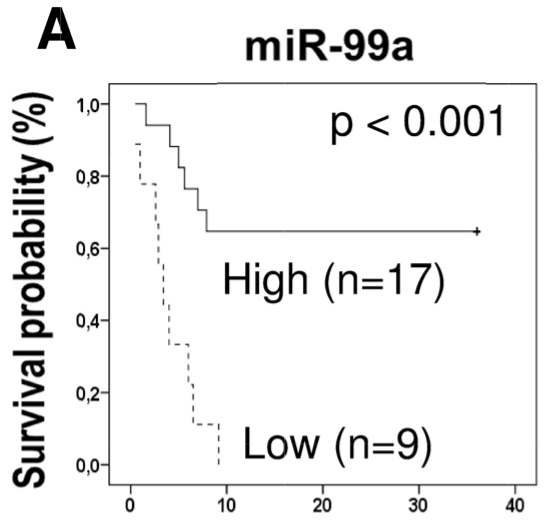

Time from diagnosis (months)

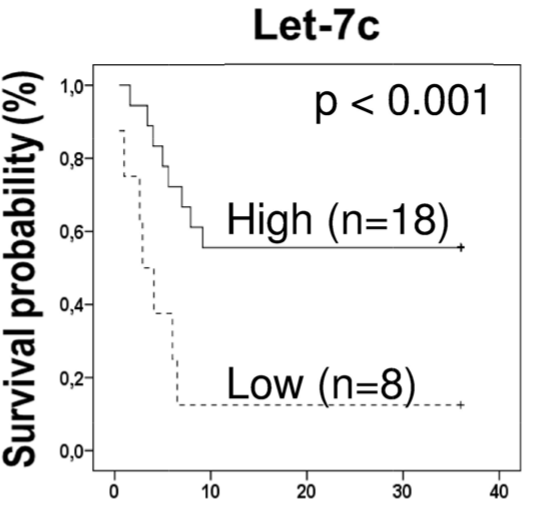

Time from diagnosis (months)

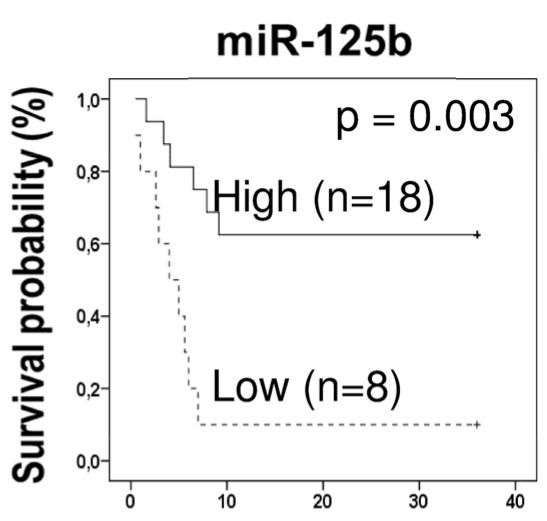

Time from diagnosis (months)

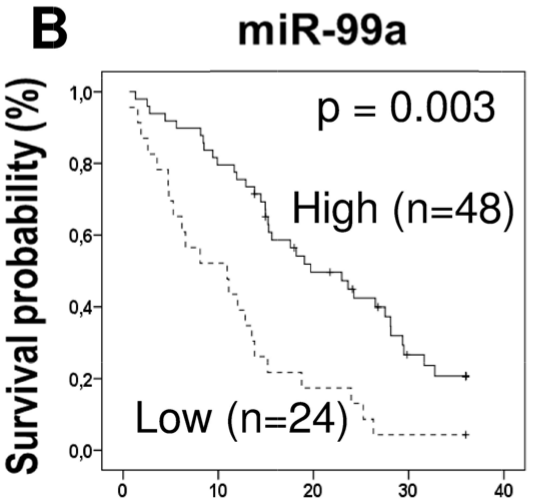

Time from diagnosis (months)
Let-7c

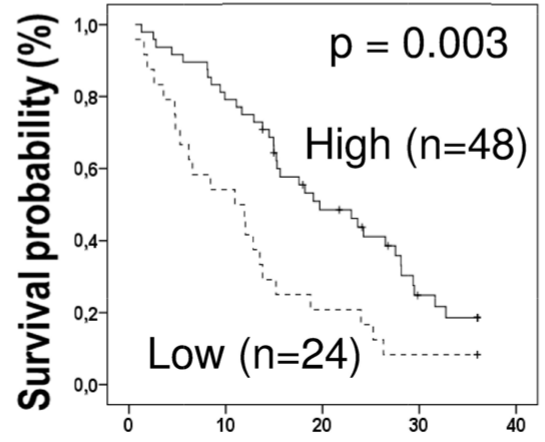

Time from diagnosis (months)

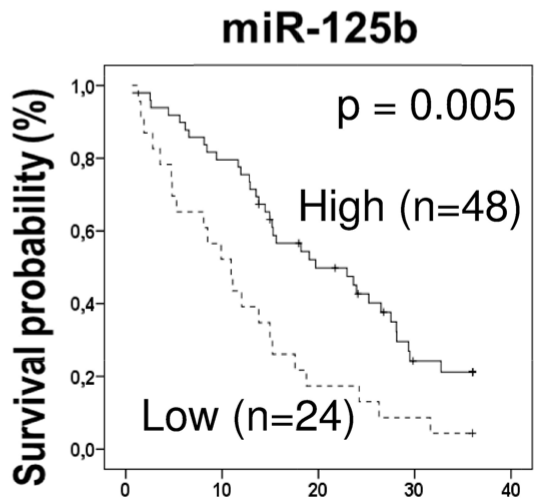

Time from diagnosis (months)

Figure 3: Prognostic value of miR-99a, let-7c, and miR-125b cluster expression in two independent cohorts of mesothelioma. Patients with tumors with low expression of these miRNAs (low third) had significantly shorter OS compared with tumors with high expression (bottom two thirds) in: (A) training set $(\mathrm{n}=26)$ and (B) TCGA set $(\mathrm{n}=72)$.

A

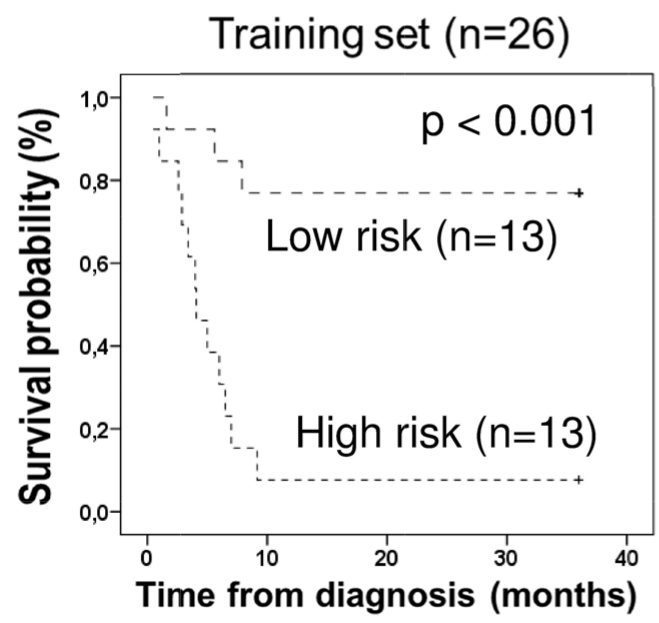

B

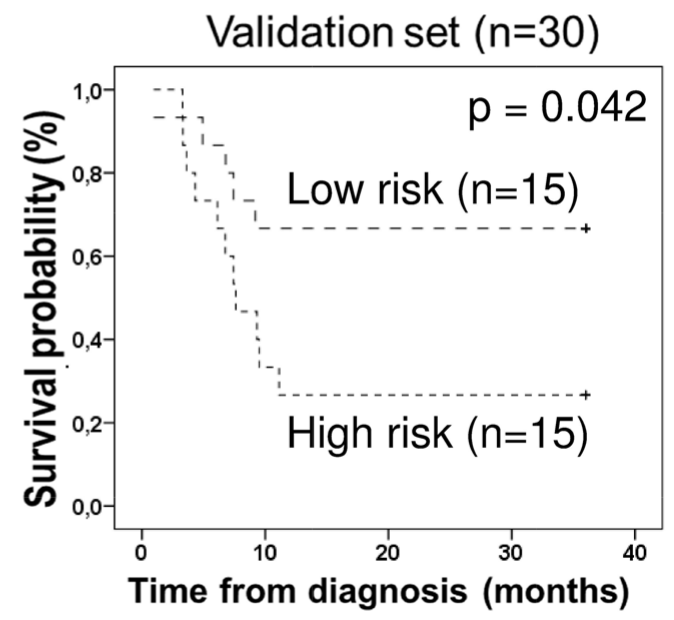

Figure 4: Kaplan-Meier plots of overall survival according to risk index based on the expression of mir-99a, let-7c, and miR-125b. Patients whose tumors had high-risk score show significantly shorter median OS as compared with patients with low-risk in two independent cohorts: (A) training set and (B) validation set. 
median risk score value. Interestingly, patients with highrisk had a shorter OS as compared to low-risk patients, and in the multivariate Cox model a high-risk score remained as an independent prognostic marker for OS.

Currently, a number of miRNAs have been correlated with prognosis in MPM patients [30-32]; in particular, Kirschner and colleagues and Lin and colleagues recently proposed the first prognostic miRNA signatures of surgically resected MPM patients [13, 32]. The lack of overlap between our miRNA signature and their results might be explained by differences in the study population and in the methods used to measure miRNA expression and to normalize the data. Specifically, Kirschner and colleagues identified a miRNA signature in a cohort of patients who underwent EPP [13]; such signature was then validated in a group of patients subjected to $\mathrm{P} / \mathrm{D}$ performed within a single institution. In contrast, our prognostic miRNA signature was developed in a cohort of unresected patients, and was identified by a high-throughput approach and then validated in two independent cohorts of patients. These differences underscore the need for building collaborative consortiums able to collect large cohorts of patients with mesothelioma to identify and validate robust markers for early diagnosis and prognosis. Overall, our results suggest that low expression of the 21q21.1 miRNA cluster could have a prognostic significance in unresected patients. However, a limitation of our study is the relatively low number of patients included in the VS mainly due to the fact that collecting samples from unresected MPM patients with long-term survival is challenging.

In conclusion, to the best of our knowledge, this is the first prognostic signature able to predict survival in patients who have not undergone surgery for MPM. Furthermore, identifying patients with different prognosis might guide clinical decisions whether to propose more aggressive or more conservative therapeutic approaches. Additionally, patients with an unfavorable miRNA signature could also benefit from novel therapeutic strategies in the future such as miRNA-based treatments, although further basic research is needed to verify the efficient translation of these approaches.

\section{MATERIALS AND METHODS}

\section{Patient enrollment}

In the present study, a total number of 27 patients diagnosed with MPM at the Istituto Nazionale per la Ricerca sul Cancro, Genova, Italy were enrolled as TS. In addition, 30 patients with clinically confirmed diagnosis of MPM were enrolled at San Andrea Hospital, La Spezia, Italy as VS. According to the clinical outcome, patients were divided into two groups: i) patients with $\mathrm{SS}$, who died within 12 months from the diagnosis (TS: 15; VS: 16) and ii) LS patients whose OS was greater than 36 months (TS: 12; VS: 14). All patients who either underwent surgery or were older than 80 years at diagnosis were not eligible and hence excluded from the study.

Four additional biopsies of nonmalignant pleura obtained from healthy individuals were processed as a normal control (NC). The non-neoplastic controls of pleural tissue were obtained from surgical specimens of lobectomy performed for non-small cell lung cancer (NSCLC, 3 adenocarcinoma and 1 squamous cell carcinoma), in which portions of parietal pleura were present. At gross examination, all NSCLCs did not show pleural invasion and histologic sections of all pleuraltissue samples were reviewed by the pathologist in order to rule out the presence of neoplastic cells.

The present study was approved by the institutional ethics committees (Istituto Nazionale per la Ricerca sul Cancro, Genova, Italy n. OT11.001, May 25, 2012 for TS; Ethics Committee of the Liguria Region P.R. 207REG2014, September 23, 2014 for VS), and conducted according to the provisions of the Declaration of Helsinki. The data were analyzed anonymously.

\section{RNA and genomic DNA (gDNA) extraction}

For each case, the FFPE biopsy tumor block was reviewed by the pathologist to check for the presence of adequate tumor cell content $(>50 \%)$. Total RNA, including the miRNA fraction, was isolated from two FFPE macro-dissected tissue sections $(10 \mu \mathrm{m})$ using the Recover All Total Nucleic Acid Isolation Kit for FFPE Tissue (ThermoFisher Scientific, Wilmington, DE, USA), including a RNase-free DNase I treatment. RNA quantity and quality were assessed by NanoDrop ND1000 (ThermoFisher Scientific) and 2100 Bioanalyzer instrument using RNA 6000 Nano Kit (Agilent Technologies, Santa Clara, CA, USA). All the samples that reported at least $150 \mathrm{ng}$ of total RNA were defined suitable for further analyses. For 44 out of 57 patients enrolled in the study, two additional tumor sections $(10 \mu \mathrm{m})$ were available to isolate gDNA using GeneRead DNA FFPE Kit (Qiagen, Hilden, Germany) that was quantified by Qubit ${ }^{\circledR}$ 2.0 Fluorometer (ThermoFisher Scientific).

\section{Microarray analysis}

MiRNA profiling was performed on $27 \mathrm{MPMs}$ from TS (15 SS and 12 LS) and 4 nonmalignant pleural samples. Total RNA containing miRNA was processed following the 'miRNA Microarray System protocol' v.2.4 (Agilent Technologies), as previously described [33]. Briefly, $100 \mathrm{ng}$ of total RNA and an appropriate dilution of Spike-in controls underwent dephosphorylation and labeling step with Cy3CTP and purification using Micro 
Bio-Spin ${ }^{\text {TM }}$ P-6 Gel Columns (Bio-Rad, Hercules, CA, USA). Then, each sample was hybridized on Human miRNA microarray slide $8 \times 60 \mathrm{~K}$ (miRBase Release 19.0) (Agilent Technologies), including 2006 human miRNAs. After washing, the slides were scanned by G2565CA scanner (Agilent Technologies) and data were extracted by Feature Extraction software v.10 (Agilent Technologies). Microarray expression data were normalized using GeneSpring software v.12.6 (Agilent Technologies). Microarray raw data have been deposited in Gene Expression Omnibus (http://www.ncbi.nlm.nih. gov/geo/; GEO number: GSE92636) [34]. Missing values corresponding to each unexpressed miRNA were filled using the minimum value and then subtracting 0.5 .

\section{Validation of selected miRNAs using publicly available data}

Clinical outcome and miRNAseq data were available from 72 patients with MPM included in the TCGA-Mesothelioma project, and these data were downloaded.

\section{Analysis of genes targeted by select miRNAs}

Validated genes targeted by select miRNAs were obtained from mirTarBase database [35]. An enriched pathway analysis was performed using DAVID functional annotation bioinformatics microarray analysis [36].

\section{Validation of selected miRNAs by qPCR}

Three selected miRNAs were then validated in a different set of tissue samples consisting of 30 patients from VS (16 SS and $14 \mathrm{LS}$ ) and $4 \mathrm{NC}$ by qPCR using TaqMan ${ }^{\circledR}$ miRNA Assays (hsa-miR-99a \#000435; hsalet-7c \#000379; hsa-miR-125b \#000449; U6 snRNA \#001973 as reference miRNA; ThermoFisher Scientific). Briefly, $10 \mathrm{ng}$ of total RNA were retro-transcripted into cDNA with a specific primer using TaqMan ${ }^{\circledR}$ MiRNA Reverse Transcription Kit (ThermoFisher Scientific). Then each selected miRNA was amplified in duplicate on RealPlex ${ }^{2}$ system (Eppendorf, Hamburg, Germany) using qPCR TaqMan ${ }^{\circledR}$ Universal Master Mix II, no Uracil-N glycoslyase (ThermoFisher Scientific) and normalized against U6 snRNA. The relative expression compared to the $\mathrm{NC}$ was determined using the equation $2^{-\Delta \Delta \mathrm{CT}}$. A patient's risk score was calculated as the sum of the expression levels of the 3 selected prognostic miRNAs in the VS, weighted by the corresponding regression coefficients $(\beta)$ derived from the Cox regression analysis in the TS, as previously reported [14]. The risk score was used to classify patients into high- or low-risk groups, with a high-risk score indicating poorer survival. In the VS, the median of the risk score was used as the cutoff value.

\section{Copy number determination of the miR-99a/let- 7c/miR-125b locus}

The CNV study of the miR-99a/let-7c/miR125b locus (21q21.1) was assessed in both TS and VS tumor samples by ddPCR according to the multiplexing strategy (three targets quantified in a triplex reaction) [37]. Specifically, custom FAM-labeled miR-99a/ let-7c/miR-125b assay (dHsaCNS694336600) was normalized with two HEX-labeled assays as follows: RBM11 (dHsaCP2506724) targeting the centromere locus on chromosome 21 and AP3B1 (dHsaCP2500348) mapping on 5q14.1 (Bio-Rad). Briefly, the ddPCR reaction including $20 \mathrm{ng}$ of gDNA, Bio-Rad ddPCR supermix for Probes No dUTP and the labeled TaqMan assays (miR-99a/let-7c/miR-125b locus assay at 100\% of concentration, RBM11 100\% and AP3B1 50\%) were converted in approximately 15,000 droplets using the QX200 Droplet Generator and amplified according to the Bio-Rad standard protocol $\left(60^{\circ} \mathrm{C}\right.$ as annealing/extension temperature). The amplified samples were then acquired by QX100 Droplet Reader and the data were analyzed using QuantaSoft ${ }^{\mathrm{TM}}$ Analysis Pro software v.1.0.596 (Bio$\mathrm{Rad}$ ). Each sample was run in duplicate with a wild type sample and negative control (no template) and the CNV of the miR-99a/let-7c/miR-125b locus was calculated as the ratio between the concentration (copies/ $\mu \mathrm{l}$ ) of the miR99a/let-7c/miR-125b locus and the mean concentration value of $R B M 11$ and $A P 3 B 1$, multiplied by 2 . Data were obtained as the result of two technical replicate wells. CNV values higher than 2.5 or lower than 1.5 were defined as amplified or deleted locus.

\section{Statistical analysis}

Class-comparison analysis between MPM/NC and $\mathrm{SS} / \mathrm{LS}$ was performed using a receiver operating characteristic (ROC) curve and the non-parametric MannWhitney U test. Survival curves were plotted using the Kaplan-Meier method, and survival differences were assessed by the log-rank test using the median of each individual miRNA as a cutoff. Univariate or multivariate Cox proportional hazards were calculated considering individual miRNA as a continuous variable. Multivariate analysis was adjusted by age and histological subtype. All calculations were performed using SPSS Statistics package $v$.15.0.

\section{Abbreviations}

CNV: copy number variation; ddPCR: droplet digital polymerase change reaction; EPP: extra-pleural pneumonectomy; FFPE: formalin-fixed paraffinembedded; gDNA: genomic DNA; LS: long survivor; MPM: malignant pleural mesothelioma; NC: normal control; NSCLC: non-small cell lung cancer; P/D: pleurectomy with decortication; qPCR: quantitative 
polymerase change reaction; SS: short survivor; TS: training Set; VS: validation set.

\section{Author contributions}

AT, SC, FG conceived and planned the study. MM, GLR, AV revised cell content of MPM samples. MGDB and IV isolated the nucleic acids and performed the quality control of miRNAs. AT and SC profiled the miRNAs by microarray and validated data by qPCR. SC and IV carried out the copy number analysis. EN and DGB analysed microarray data. AT, SC, EN, CG contributed to data interpretation and wrote the manuscript. AA, ER, FB, GB contributed to data interpretation and revised manuscript. PF, AV, FF, SR enrolled and analysed patients for the validation set. DFM and SR recovered all clinical data of patients. SS setup the workflow of miRNA isolation from FFPE tissue. All authors discussed the results, reviewed and approved the manuscript.

\section{ACKNOWLEDGMENTS}

We kindly thank Dr. Cristina Bruzzo and Dr. Zita Cavalieri (Lung Cancer Unit, Ospedale Policlinico San Martino, Genoa, Italy) for their technical support.

\section{CONFLICTS OF INTEREST}

The authors declare no conflicts of interest.

\section{FUNDING}

$\mathrm{SC}$ is a $\mathrm{PhD}$ supported by the Italian Ministry of Health (GR-2011-02350922), EN is supported by a Juan Rodés Scholarship from the Carlos III Health Institute (JR13/00002). This study was supported by grants from Italian Ministry of Health (5 x 1000 funds 2013; FG) and Università degli Studi di Torino, Italy (D85E12003020001; FG). In addition, the study was partially supported by CERCA and the Instituto de Salud Carlos III (PI14/01109) co-funded by FEDER funds-a way to build Europe (EN).

\section{REFERENCES}

1. Robinson BW, Lake RA. Advances in malignant mesothelioma. N Engl J Med. 2005; 353:1591-1603.

2. van der Bij S, Koffijberg H, Burgers JA, Baas P, van de Vijver MJ, de Mol BA, Moons KG. Prognosis and prognostic factors of patients with mesothelioma: a population-based study. Br J Cancer. 2012; 107:161-164.

3. Maggioni C, Barletta G, Rijavec E, Biello F, Gualco E, Grossi F. Advances in treatment of mesothelioma. Expert Opin Pharmacother. 2016; 17:1197-1205.
4. Lim E. MARS 2: A Feasibility Study Comparing (Extended) Pleurectomy Decortication Versus no Pleurectomy Decortication in Patients With Malignant Pleural Mesothelioma (MARS2). NCT02040272. Available at: https://clinicaltrials.gov/ct2/show/NCT02040272 Date last updated: April 12 2016. Date last accessed: December 52016.

5. Vogelzang NJ, Rusthoven JJ, Symanowski J, Denham C, Kaukel E, Ruffie P, Gatzemeier U, Boyer M, Emri S, Manegold C, Niyikiza C, Paoletti P. Phase III study of pemetrexed in combination with cisplatin versus cisplatin alone in patients with malignant pleural mesothelioma. J Clin Oncol. 2003; 21:2636-2644.

6. Zalcman G, Mazieres J, Margery J, Greillier L, AudigierValette C, Moro-Sibilot D, Molinier O, Corre R, Monnet I, Gounant V, Rivière F, Janicot H, Gervais R, et al. Bevacizumab for newly diagnosed pleural mesothelioma in the Mesothelioma Avastin Cisplatin Pemetrexed Study (MAPS): a randomised, controlled, open-label, phase 3 trial. Lancet. 2016; 387:1405-1414.

7. Guled M, Lahti L, Lindholm PM, Salmenkivi K, Bagwan I, Nicholson AG, Knuutila S. CDKN2A, NF2, and JUN are dysregulated among other genes by miRNAs in malignant mesothelioma-A miRNA microarray analysis. Genes Chromosomes Cancer. 2009; 48:615-623.

8. Balatti V, Maniero S, Ferracin M, Veronese A, Negrini M, Ferrocci G, Martini F, Tognon MG. MicroRNAs dysregulation in human malignant pleural mesothelioma. J Thorac Oncol. 2011; 6:844-851.

9. Benjamin H, Lebanony D, Rosenwald S, Cohen L, Gibori H, Barabash N, Ashkenazi K, Goren E, Meiri E, Morgenstern S, Perelman M, Barshack I, Goren Y, et al. A diagnostic assay based on microRNA expression accurately identifies malignant pleural mesothelioma. J Mol Diagn. 2010; 12:771-779.

10. Busacca S, Germano S, De Cecco L, Rinaldi M, Comoglio F, Favero F, Murer B, Mutti L, Pierotti M, Gaudino G. MicroRNA signature of malignant mesothelioma with potential diagnostic and prognostic implications. Am J Respir Cell Mol Biol. 2010; 42:312-319.

11. Gee GV, Koestler DC, Christensen BC, Sugarbaker DJ, Ugolini D, Ivaldi GP, Resnick MB, Houseman EA, Kelsey KT, Marsit CJ. Downregulated microRNAs in the differential diagnosis of malignant pleural mesothelioma. Int J Cancer. 2010; 127:2859-2869.

12. Pass HI, Goparaju C, Ivanov S, Donington J, Carbone M, Hoshen M, Cohen D, Chajut A, Rosenwald S, Dan H, Benjamin S, Aharonov R. hsa-miR-29c* is linked to the prognosis of malignant pleural mesothelioma. Cancer Res. 2010; 70:1916-1924.

13. Kirschner MB, Cheng YY, Armstrong NJ, Lin RC, Kao SC, Linton A, Klebe S, McCaughan BC, van Zandwijk N, Reid G. MiR-score: a novel 6-microRNA signature that predicts 
survival outcomes in patients with malignant pleural mesothelioma. Mol Oncol. 2015; 9:715-726.

14. Chen HY, Yu SL, Chen CH, Chang GC, Chen CY, Yuan A, Cheng CL, Wang CH, Terng HJ, Kao SF, Chan WK, Li HN, Liu CC, et al. A five-gene signature and clinical outcome in non-small-cell lung cancer. N Engl J Med. 2007; 356:11-20.

15. Walter RF, Vollbrecht C, Werner R, Wohlschlaeger J, Christoph DC, Schmid KW, Mairinger FD. microRNAs are differentially regulated between MDM2-positive and negative malignant pleural mesothelioma. Oncotarget. 2016; 7:18713-21. https://doi.org/10.18632/oncotarget.7666.

16. Yamada H, Yanagisawa K, Tokumaru S, Taguchi A, Nimura Y, Osada H, Nagino M, Takahashi T. Detailed characterization of a homozygously deleted region corresponding to a candidate tumor suppressor locus at 21q11-21 in human lung cancer. Genes Chromosomes Cancer. 2008; 47:810-18. https://doi.org/10.1002/ gcc. 20582.

17. Chen Z, Jin Y, Yu D, Wang A, Mahjabeen I, Wang C, Liu X, Zhou X. Downregulation of the microRNA-99 family members in head and neck squamous cell carcinoma. Oral Oncol. 2012; 48:686-691.

18. Xin JX, Yue Z, Zhang S, Jiang ZH, Wang PY, Li YJ, Pang M, Xie SY. miR-99 inhibits cervical carcinoma cell proliferation by targeting TRIB2. Oncol Lett. 2013; 6:1025-1030.

19. Forés-Martos J, Cervera-Vidal R, Chirivella E, RamosJarero A, Climent J. A genomic approach to study down syndrome and cancer inverse comorbidity: untangling the chromosome 21. Front Physiol. 2015; 6:10.

20. Wu D, Zhou Y, Pan H, Qu P, Zhou J. microRNA-99a inhibits cell proliferation, colony formation ability, migration and invasion by targeting fibroblast growth factor receptor 3 in prostate cancer. Mol Med Rep. 2015; 11:1469-1475.

21. Nadiminty N, Tummala R, Lou W, Zhu Y, Zhang J, Chen X, deVere White RW, Kung HJ, Evans CP, Gao AC. MicroRNA let-7c suppresses androgen receptor expression and activity via regulation of Myc expression in prostate cancer cells. J Biol Chem. 2012; 287:1527-1537.

22. Johnson SM, Grosshans H, Shingara J, Byrom M, Jarvis R, Cheng A, Labourier E, Reinert KL, Brown D, Slack FJ. RAS is regulated by the let-7 microRNA family. Cell. 2005; 120:635-647.

23. Peng SC, Liao CT, Peng CH, Cheng AJ, Chen SJ, Huang CG, Hsieh WP, Yen TC. MicroRNAs MiR-218, MiR-125b, and Let-7g predict prognosis in patients with oral cavity squamous cell carcinoma. PLoS One. 2014; 9:e102403.

24. Ying X, Wei K, Lin Z, Cui Y, Ding J, Chen Y, Xu B. MicroRNA-125b Suppresses Ovarian Cancer Progression via Suppression of the Epithelial-Mesenchymal Transition Pathway by Targeting the SET Protein. Cell Physiol Biochem. 2016; 39:501-510.
25. Sun D, Layer R, Mueller AC, Cichewicz MA, Negishi M, Paschal BM, Dutta A. Regulation of several androgeninduced genes through the repression of the miR-99a/ let-7c/miR-125b-2 miRNA cluster in prostate cancer cells. Oncogene. 2014; 33:1448-1457.

26. Lin KY, Ye H, Han BW, Wang WT, Wei PP, He B, Li XJ, Chen YQ. Genome-wide screen identified let-7c/miR99a/miR-125b regulating tumor progression and stemlike properties in cholangiocarcinoma. Oncogene. 2016; 35:3376-3386

27. Linton A, van Zandwijk N, Reid G, Clarke S, Cao C, Kao $\mathrm{S}$. Inflammation in malignant mesothelioma - friend or foe? Ann Cardiothorac Surg. 2012; 1:516-522.

28. Abdul Rahim SN, Ho GY, Coward JI. The role of interleukin-6 in malignant mesothelioma. Transl Lung Cancer Res. 2015; 4:55-66.

29. Nižetić D, Groet J. Tumorigenesis in Down's syndrome: big lessons from a small chromosome. Nat Rev Cancer. 2012; 12:721-732.

30. Truini A, Coco S, Alama A, Genova C, Sini C, Dal Bello MG, Barletta G, Rijavec E, Burrafato G, Boccardo F, Grossi F. Role of microRNAs in malignant mesothelioma. Cell Mol Life Sci. 2014; 71:2865-2878.

31. Truini A, Coco S, Genova C, Mora M, Dal Bello MG, Vanni I, Alama A, Rijavec E, Barletta G, Biello F, Maggioni C, Grossi F. Prognostic and Therapeutic Implications of MicroRNA in Malignant Pleural Mesothelioma. MicroRNA. 2016; 5:12-18.

32. Lin RC, Kirschner MB, Cheng YY, van Zandwijk N, Reid G. MicroRNA gene expression signatures in long-surviving malignant pleural mesothelioma patients. Genom Data. 2016; 9:44-49.

33. Scaruffi P, Stigliani S, Moretti S, Coco S, De Vecchi C, Valdora F, Garaventa A, Bonassi S, Tonini GP. TranscribedUltra Conserved Region expression is associated with outcome in high-risk neuroblastoma. BMC Cancer. 2009; 9:441.

34. Truini A, Coco S, Nadal E. Down modulation of miR-99a/ let-7c/miR-125b miRNA cluster predicts clinical outcome in patients with unresected malignant pleural mesothelioma. Gene Expression Omnibus. 2017; GSE92636.

35. Chou $\mathrm{CH}$, Chang NW, Shrestha S, Hsu SD, Lin YL, Lee WH, Yang CD, Hong HC, Wei TY, Tu SJ, Tsai TR, Ho SY, Jian TY, et al. miRTarBase 2016: updates to the experimentally validated miRNA-target interactions database. Nucleic Acids Res. 2016; 44:D239-47.

36. Huang DW, Sherman BT, Lempicki RA. Systematic and integrative analysis of large gene list using DAVID bioinformatics resources. Nat Protoc. 2009; 4:44-57.

37. Whale AS, Huggett JF, Tzonev S. Fundamentals of multiplexing with digital PCR. Biomol Detect Quantif. 2016; 10:15-23. 